\title{
Altered exhaled biomarker profiles in children during and after rhinovirus- induced wheeze
}

\author{
Marc P. van der Schee ${ }^{1,2,3}$, Simone Hashimoto ${ }^{1}$, Annemarie C. Schuurman ${ }^{1}$, \\ Janine S. Repelaer van Driel ${ }^{1}$, Nora Adriaens ${ }^{1}$, Romy M. van Amelsfoort ${ }^{1}$, \\ Tessa Snoeren ${ }^{1}$, Martine Regenboog ${ }^{1}$, Aline B. Sprikkelman², Eric G. Haarman ${ }^{3}$, \\ Wim M.C. van Aalderen ${ }^{2}$ and Peter J. Sterk ${ }^{1}$ \\ Affiliations: ${ }^{1}$ Dept of Respiratory Medicine, Academic Medical Centre, University of Amsterdam, Amsterdam, \\ The Netherlands. ${ }^{2}$ Dept of Pediatric Respiratory Medicine and Allergy, Emma's Children Hospital, Academic \\ Medical Centre, University of Amsterdam, Amsterdam, The Netherlands. ${ }^{3}$ Dept of Pediatric Respiratory \\ Medicine, VU Medical Centre, VU University of Amsterdam, Amsterdam, The Netherlands.
}

Correspondence: M.P. van der Schee, Dept of Respiratory Medicine, F5-158, Academic Medical Centre, University of Amsterdam, Meibergdreef 9, 1105 AZ Amsterdam, The Netherlands.

E-mail: m.p.vanderscheedamc.uva.nl

ABSTRACT Preschool rhinovirus-induced wheeze is associated with an increased risk of asthma. In adult asthma, exhaled volatile organic compounds (VOC) are associated with inflammatory activity. We therefore hypothesised that acute preschool wheeze is accompanied by a differential profile of exhaled VOC, which is maintained after resolution of symptoms in those children with rhinovirus-induced wheeze.

We included 178 children (mean \pm SD age $22 \pm 9$ months) from the EUROPA cohort comparing asymptomatic and wheezing children during respiratory symptoms and after recovery. Naso- and oropharyngeal swabs were tested for rhinovirus by quantitative PCR. Breath was collected via a spacer and analysed using an electronic nose. Between-group discrimination was assessed by constructing a 1000-fold cross-validated receiver operating characteristic curve. Analyses were stratified by rhinovirus presence/absence.

Wheezing children demonstrated a different VOC profile when compared with asymptomatic children $(\mathrm{p}<0.001$ ), regardless of the presence (area under the curve (AUC) 0.77, 95\% CI 0.07) or absence (AUC $0.81,95 \%$ CI 0.05 ) of rhinovirus. After symptomatic recovery, discriminative accuracy was maintained in children with rhinovirus-induced wheeze (AUC 0.84, 95\% CI 0.06), whereas it dropped significantly in infants with non-rhinovirus-induced wheeze (AUC 0.67, 95\% CI 0.06).

Exhaled molecular profiles differ between preschool children with and without acute respiratory wheeze. This appears to be sustained in children with rhinovirus-induced wheeze after resolution of symptoms. Therefore, exhaled VOC may qualify as candidate biomarkers for early signs of asthma.

@ERSpublications

Children with rhinovirus-induced wheeze have altered exhaled biomarkers both during symptoms and after resolution http://ow.ly/C6vWT

\footnotetext{
This article has supplementary material available from erj.ersjournals.com

Received: March 072014 | Accepted after revision: Sept 032014 | First published online: Oct 162014

Support statement: This study was funded by the Dutch Asthma Foundation and Biomerieux, France.

Conflict of interest: Disclosures can be found alongside the online version of this article at erj.ersjournals.com
}

Copyright OERS 2015 


\section{Introduction}

About one in three children has lower respiratory tract symptoms (LRTS) resembling the symptoms of asthma during the first 3 years of life [1]. About one-third of these children will eventually be diagnosed with asthma at school age [2]. Virtually all episodes of respiratory symptoms can be linked to viral infections [3]. Amongst these, rhinovirus-induced LRTS may promote an exaggerated inflammatory response, possibly promoting the development of asthmatic features [4]. This may explain their strong association with a subsequent diagnosis of school-age asthma $[5,6]$, suggesting that rhinovirus-induced wheeze represents a phenotype of preschool wheeze with prognostic relevance.

Nonetheless, accurate prediction of asthma in wheezy children remains challenging. Current symptom-based predictive rules generally have limited accuracy due to heterogeneity of the disease processes underlying LRTS in infancy [7]. Assessing more objective criteria, such as airway hyperresponsiveness, airway obstruction or airway inflammation, is challenging, if not impossible, to perform reliably in preschool children. This creates a need for minimally invasive biomarkers reflecting the pathobiology underlying wheeze and, potentially, the development of asthma in children.

Along these lines, analysis of breath-based biomarkers, such as exhaled nitric oxide fraction ( FeNO), have received considerable attention. FeNO has been shown to be elevated with increased airway responsiveness [8] and to aid in the prediction of asthma symptoms in preschool wheeze [9]. Unfortunately, its predictive value is hampered by the heterogeneity of conditions altering FeNO values [10]. Given the complexity of the histological abnormalities that are already manifest in bronchial biopsies of preschool wheezers [11], composite biomarkers may more robustly reflect the multifactorial pathophysiology of the disease processes underlying wheeze and its potential progression towards asthma [7]. This can be approached by high-throughput "omics" technologies [12].

A noninvasive omics approach is the analysis of exhaled volatile organic compounds (VOC). In adults, several studies have independently shown that VOC can differentiate between patients with asthma, patients with chronic obstructive pulmonary disease and healthy controls [13, 14]. In children with established asthma, volatile biomarker analysis enabled prediction of subsequent exacerbations [15]. Furthermore, a recent study by VAN DE KANT et al. [16] showed that children with preschool wheeze have different VOC when compared with their healthy counterparts. These results are likely to be driven by the association of VOC with inflammatory markers such as eosinophilia $[17,18]$. This raises the possibility that a prolonged inflammatory response associated with rhinovirus-induced wheeze could be detected by VOC analysis.

Therefore, we first hypothesised that acute preschool wheeze is reflected by a differential profile of exhaled VOC. Second, we hypothesised that this VOC profile is maintained after resolution of symptoms in those children with rhinovirus-induced wheeze. Our aim was to study this by assessing exhaled VOC in asymptomatic and wheezing children during the acute episode and after symptomatic recovery. All the analyses were stratified according to the presence/absence of rhinovirus to assess specificity of our outcomes for rhinoviral infections. By doing so, we aimed to establish whether exhaled biomarkers have potential for the assessment of a high-risk phenotype in the development of asthma.

\section{Methods}

Subjects

This study is part of the Early Unbiased Risk Assessment of Pediatric Asthma (EUROPA) trial, a prospective cohort study aimed at early prediction of asthma. Subjects were recruited by targeted mailing from an unselected birth cohort of 12033 children born in greater Amsterdam, aged between 0 and 12 months at inclusion. Infants were eligible if not born under 31 weeks of gestation and were known not to have any manifest illness, specifically any pulmonary disorder. 1216 infants were included into the trial after both parents provided consent (fig. 1). At inclusion, a structured baseline questionnaire was obtained as modified from the EuroPrevall study on pregnancy, family history and general health [19].

\section{Design}

This study was designed as a prospective nested case-control follow-up cohort study. The cohort was monitored for the occurrence of acute episodes of LRTS from December 2010 to December 2012. When parents called the research centre, a structured telephone interview was obtained. Children experiencing cough, wheezing and/or dyspnoea sufficiently severe for parents to warrant a visit to their family physician entered the symptomatic arm of the study, and were visited within $8 \mathrm{~h}$ of establishing these symptoms. At that time, a structured questionnaire including the Pediatric Respiratory Assessment Measure (PRAM)and Asthma Control Questionnaire (ACQ)-based severity score [20, 21] were administered (realising that the ACQ has not been validated for this age group). Exhaled breath was analysed, and naso- and oropharyngeal swabs were collected for viral diagnosis. A trained researcher performed auscultation to 


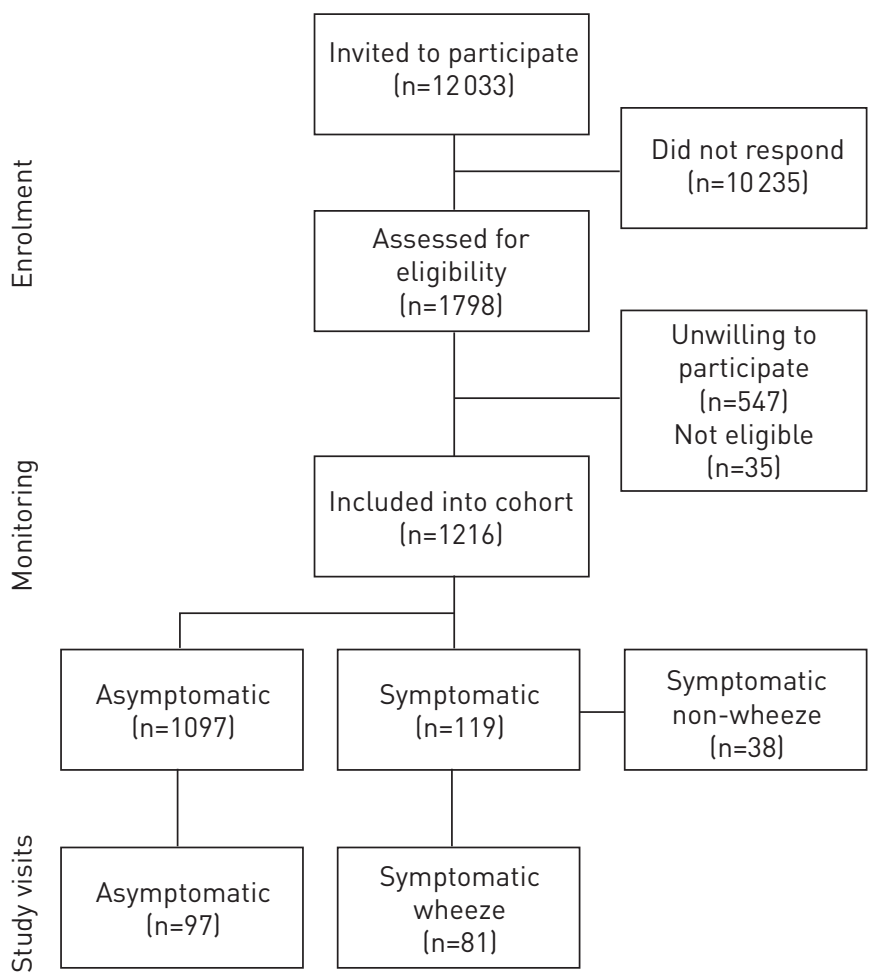

FIGURE 1 Consolidated Standards of Reporting Trials diagram for this study detailing the number of subjects enrolled, monitored and studied.

determine the presence or absence of wheeze. Children with confirmed expiratory wheeze were scheduled to be re-assessed a minimum of 6 weeks later after a symptom-free episode of $\geqslant 1$ week, defined as a PRAM- and ACQ-based severity score of 0 . At that time point, all aforementioned measurements were repeated. Children in whom wheeze could not be confirmed were re-assessed upon recurrence of their symptoms to determine whether they should be scheduled for a visit after recovery of these symptoms. Only one symptomatic visit was included in the study for each child.

Asymptomatic children were studied as controls. These children were recruited by random selection from the same cohort and had never experienced LRTS severe enough to contact their family physician. If LRTS occurred after inclusion as a control, a new control was recruited. Regardless of the presence of symptoms, all parents were requested to fill out a bi-annual International Study of Asthma and Allergies in Childhood-based questionnaire [22], matched to their birth date, to monitor respiratory symptoms and the general health of children. This study was approved by the Medical Ethical Committee of the Academic Medical Centre Amsterdam, Amsterdam, the Netherlands (approval number 09/066) and the parents gave written informed consent. The EUROPA study is registered in the Dutch Trial Register (NTR-1955).

Viral diagnosis

All naso- and oropharyngeal swabs (Copan, Brescia, Italy) were tested within $6 \mathrm{~h}$ of collection for the presence of 14 respiratory viruses by multiplex quantitative PCR (rhinovirus, respiratory syncytial virus, influenza viruses A and B, enterovirus, metapneumovirus, coronavirus, parechovirus, parainfluenza viruses 1-4, bocavirus, and adenovirus) [23]. A virus was deemed present when either the naso- or oropharyngeal swab tested positive.

\section{Breath collection and analysis}

Exhaled breath was collected by means of a Babyhaler (GlaxoSmithKline, Brentford, UK) with inverted valve system. Children breathed tidally into a face mask covering the mouth and nose. Environmental VOC inside the child's airways and the spacer were washed out during $100 \mathrm{~s}$ of breathing through an inspiratory VOC filter (North Safety Products, Middelburg, the Netherlands). Ambient air drawn through an identical VOC filter was used to create a baseline reference signal. The exhaled breath was subsequently sampled for $40 \mathrm{~s}$ directly from the spacer by two separate Cyranose 320 Electronic Nose (eNose) devices (IOS Inc., Pasadena, CA, USA). A Cyranose 320 has an array of 32 nano-sensors that show nonselective interactions with the VOC mixture. This creates a so-called breathprint, a pattern of resistance changes for 
the individual sensors that reflects the profile of the VOC mixture. This device has commonly been used for breath analysis, and has shown adequate repeatability and reproducibility [24]. Further details on methodology are available in the online supplementary material.

\section{Sample size estimation}

Based on a pilot study comparing children with and without expiratory wheeze, we established that a sample size of 21 subjects per group would be sufficient to reject the null hypothesis that VOC analysis cannot discriminate symptomatic and asymptomatic children with a power of $90 \%$. Additional information on this pilot study is available in the online supplementary material.

\section{Data analysis}

Data were analysed using SPSS (version 22.0; IBM, Armonk, NY, USA). Subject characteristics were compared by independent t-tests or the Chi-squared test when applicable. The variance of the original breathprints was recombined into a set of six orthogonal factors by principal component analysis (based on capturing a variance of $\sim 99 \%$ with factor loadings $>0.1$ ). The discriminative potential of these factors was assessed by independent t-test for between-subject analysis and dependent t-test for within-subject testing. Discriminating principal components $(\mathrm{p}<0.05)$ were subsequently used in a canonical discrimination analysis. This technique creates an algorithm that provides the probability for each subject that it is a case or control. These probabilities were used to construct receiver operator characteristic (ROC) curves with areas under the curve (AUCs), sensitivities, specificities, and negative and positive likelihood ratios.

Data were validated by creating a random training and validation set in a $2 / 1$ ratio. In order to check the reliability of the outcomes, the AUC was determined by generating 1000 random classifications of the subjects into training and validation sets, and calculating the mean AUC for these random distributions and their associated performance characteristics [25]. Single-point sensitivity and specificity values were drawn from the AUC curves to illustrate the model characteristics based on the Youden index. A p-value of $<0.05$ was considered statistically significant. Replicate analysis obtained by the second eNose is presented separately. The intraclass correlation coefficient and Crohnbach's alpha were calculated to assess the between-device reproducibility of AUCs. Potential confounding of our results by use of inhaled corticosteroids (ICS) or $\beta_{2}$-agonists in the symptomatic group was assessed by comparing VOC profiles of symptomatic children with and without such medication. The effect of age on the discriminating principal components was assessed within noninfected healthy controls.

\section{Results}

178 children participated in the study (fig. 1). Symptomatic children were significantly younger than asymptomatic children for both rhinovirus-positive and -negative cases. Asymptomatic children had a positive PCR for any of the viruses in 39\% of the cases. For symptomatic children, this proportion was $83 \%(\mathrm{p}<0.001)$. Rhinovirus-negative symptomatic children had significantly fewer co-infections then did rhinovirus-positive symptomatic children $(\mathrm{p}<0.05)$. Detailed baseline characteristics are presented in table 1.

\section{Association between acute wheeze and exhaled breathprint}

Six principal components captured $99.7 \%$ of the original variance for rhinovirus-positive children, of which three principal components significantly differentiated between symptomatic and asymptomatic children. For rhinovirus-negative children, $99.6 \%$ of the variance was captured, of which two components differentiated between the two target groups.

In the training set, the constructed algorithm differentiated between children with acute respiratory wheeze and asymptomatic children with respect to their exhaled VOC profile, regardless of the presence (AUC $0.86,95 \%$ CI $0.10 ; \mathrm{p}<0.001$ ) or absence (AUC $0.87,95 \%$ CI $0.07 ; \mathrm{p}<0.001$ ) of rhinovirus. In the validation set, the accuracies were AUC 0.77 (95\% CI 0.07; p<0.001) and AUC 0.81 (95\% CI 0.05; p<0.001) for rhinovirus-positive and -negative children, respectively. A ROC curve for the latter algorithm stratified according to the presence of rhinovirus is presented in figure 2.

\section{Breathprint of rhinovirus-induced wheeze after recovery}

Six principal components were constructed to capture $99.1 \%$ of the original variance for rhinovirus positive and $99.6 \%$ of the variance for rhinovirus-negative cases. Three of these principal components differentiated between recovered children and controls for rhinovirus-positive children, and two for rhinovirus-negative children. Upon recovery of symptoms, the exhaled VOC profile of children with rhinovirus-induced wheeze could still be discriminated with a similar accuracy from that of asymptomatic children in the training (AUC 0.87, 95\% CI 0.10; p<0.001) and validation set (AUC 0.84, 95\% CI 0.06; 
TABLE 1 Baseline subject characteristics

\begin{tabular}{|c|c|c|c|c|}
\hline & \multicolumn{2}{|c|}{ Rhinovirus positive } & \multicolumn{2}{|c|}{ Rhinovirus negative } \\
\hline Subjects $\mathrm{n}$ & 37 & 26 & 44 & 71 \\
\hline Age at first visit months & $16.8 \pm 9.4$ & $27.8 \pm 4.9 * *$ & $19.5 \pm 9.4$ & $28.3 \pm 3.5^{* *}$ \\
\hline Birth weight kg & $3.5 \pm 0.6$ & $3.6 \pm 0.6$ & $3.5 \pm 0.6$ & $3.5 \pm 0.5$ \\
\hline Prematurity & 1 (3) & $0(0)$ & $0(0)$ & 5 (7) \\
\hline Prenatal smoke exposure & $1(3)$ & $1(4)$ & $4(9)$ & $3(4)$ \\
\hline Environmental smoke exposure ${ }^{\#}$ & $4(11)$ & $3(12)$ & $7(16)$ & $5(7)$ \\
\hline$\beta_{2}$-agonist use & $23(62)$ & NA & $25(57)$ & NA \\
\hline ICS use & $9(24)$ & NA & $11(25)$ & NA \\
\hline PRAM severity & $2.5 \pm 2.0$ & NA & $2.4 \pm 1.7$ & NA \\
\hline ACQ-based control & $14.6 \pm 7.3$ & NA & $16.9 \pm 6.5$ & NA \\
\hline
\end{tabular}

Data are presented as $\mathrm{n}(\%)$ or mean $\pm \mathrm{SD}$, unless otherwise stated. Breastfed infants were breastfed at least during the first month of their life. Parental asthma was defined as a parent-reported doctor diagnosis of asthma in either parent. Prenatal smoke exposure was defined as smoking by or near the mother while she was pregnant. ICS: inhaled corticosteroid; PRAM: Pediatric Respiratory Assessment Measure; ACQ: Asthma Control Questionnaire; NA: not applicable. \#: missing values ( $n=2)$ were replaced by the respective group mean. *: $p<0.05$ for comparison with symptomatic children; ${ }^{* *}: p<0.01$ for comparison with symptomatic children.

$\mathrm{p}<0.001)$. By contrast, the accuracy of this discrimination decreased significantly $(\mathrm{p}<0.05)$ after resolution of symptoms in children with non-rhinovirus-induced wheeze (training: AUC 0.71 (95\% CI 0.10; $\mathrm{p}<0.001)$; validation: AUC 0.67 (95\% CI 0.06; $\mathrm{p}=0.005)$ ). A ROC curve for the validation set algorithm, stratified according to the presence of rhinovirus, is presented in figure 3. Detailed performance characteristics for validation algorithms are presented in table 2. The training set algorithms are detailed in online supplementary table E1.

\section{Confounders and replicate analyses}

We did not find statistically significant differences after stratification for the use of ICS ( $p=0.11)$ or the use of $\beta_{2}$-agonists $(\mathrm{p}=0.07)$. However, influence of concomitant medication cannot be fully excluded and requires further study. Furthermore, age did not affect the VOC profile $(p>0.13)$. These results were reproduced by a second eNose with similar accuracies. There were no significant differences between the accuracies of the devices for any of the analyses. The intraclass correlation coefficient for eNoses 1 and 2 was 0.85 with a Crohnbach's alpha of 0.83 , indicating good reproducibility. Details of performance characteristics for replicate analyses are available in the online supplementary material for both training (table E3) and validation datasets (table E2)

\section{Discussion}

Our study has shown that children with LRTS have significantly different exhaled VOC profiles compared with asymptomatic children, regardless of the presence or absence of rhinovirus. Consistent with this, we found that the accuracy of this discrimination decreases significantly after children with non-rhinovirus-induced wheeze recovered from their infection. However, despite full symptomatic recovery exhaled biomarkers in children with rhinovirus-induced wheeze could still be discriminated with similar accuracy from asymptomatic children. These findings indicate that a prolonged (inflammatory) response associated with symptomatic rhinovirus infections may be captured by exhaled VOC. As rhinovirus-induced wheeze is associated with a 10-fold increased risk of a future diagnosis of asthma [6], this study suggests that exhaled VOC are candidate biomarkers to noninvasively detect early signs of asthma in preschool children. This warrants long-term, prospective follow-up.

To our knowledge, this is the first study addressing exhaled biomarkers in relation to viral presence in children with LRTS. The present results thereby extend those of VAN DE KANT et al. [16], who showed that VOC profiles can distinguish children with and without wheeze during asymptomatic episodes with a modest sensitivity of $79 \%$ and a low specificity of $50 \%$. Their analysis resembles the present comparison of 


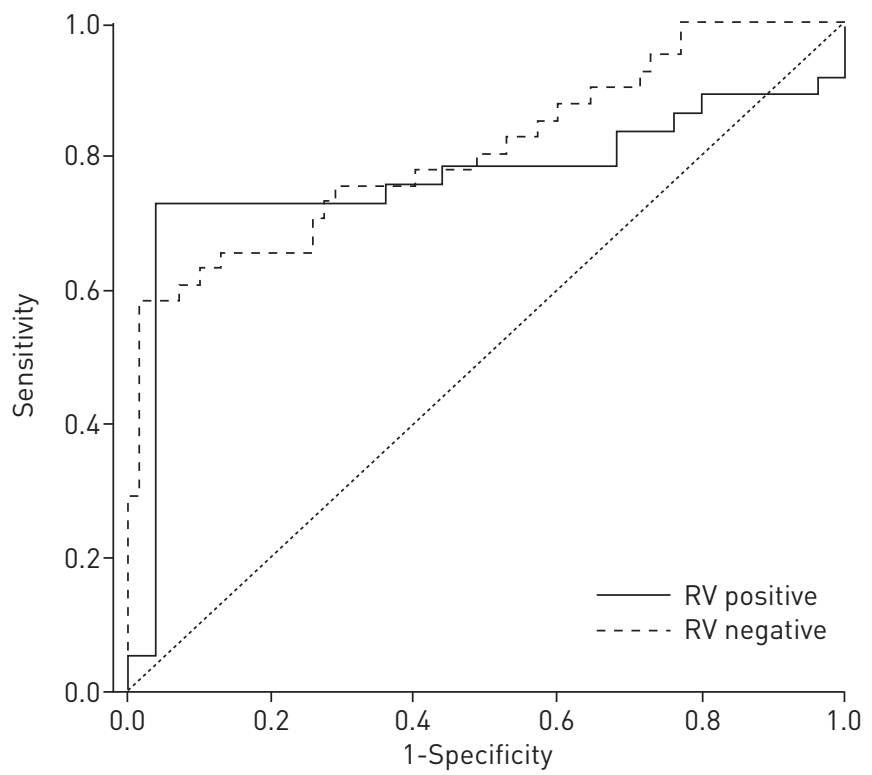

FIGURE 2 Receiver operating characteristic curves for discrimination of symptomatic and asymptomatic children by volatile organic compound analysis in rhinovirus (RV)-positive and -negative cases in the validation dataset. RV positive: area under the curve (AUC) 0.77 (95\% CI 0.07; p<0.001); sensitivity $73.0 \%$; specificity $96.0 \%$. RV negative: AUC 0.81 (95\% CI $0.05 ; \mathrm{p}<0.001)$; sensitivity $75.6 \%$; specificity $71.4 \%$.

symptomatic children after resolution of symptoms with those who were asymptomatic. In fact, our data may help to explain the relatively limited specificity in the study by VAN DE KANT et al. [16], showing that this distinction is dependent on the presence or absence of a rhinovirus during the acute episode.

We made large efforts to ensure the validity of our findings by careful methodological choices. A strength of the study is the large, well-characterised and unselected birth cohort, which allowed prospective monitoring of LRTS. The criteria for symptomatic children and their re-evaluation after recovery were selected in order to mimic the real-life population of a general practitioners office. This was meant to maximise external validity in relation to first-line medicine. The validity was further increased by a stringent statistical approach for discovery of molecular signatures $[25,26]$, which validated the results by

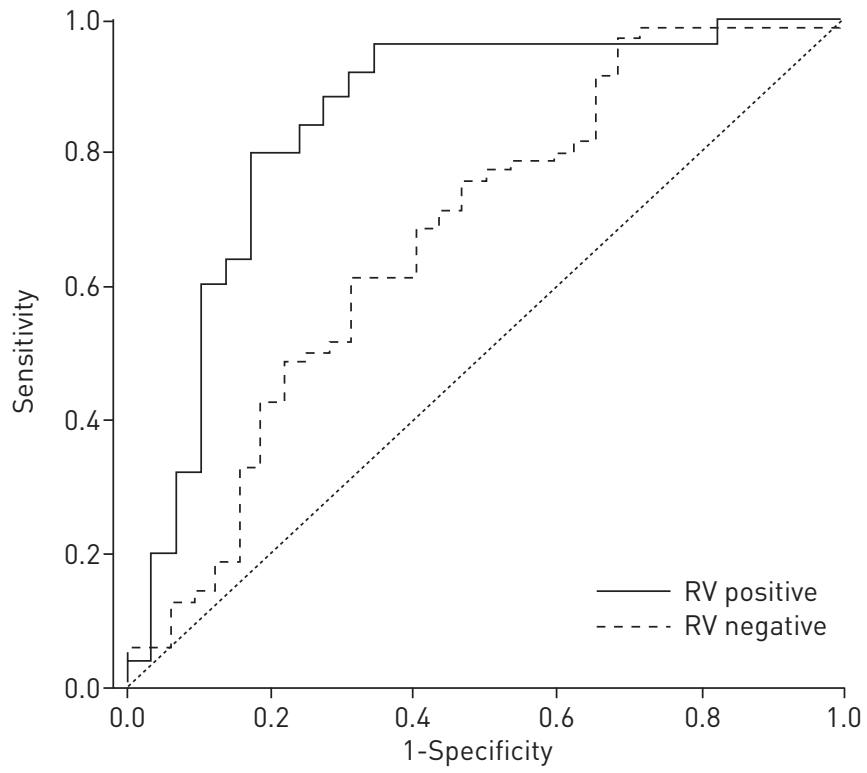

FIGURE 3 Receiver operating characteristic curves for discrimination of recovered former symptomatic and asymptomatic children by volatile organic compound analysis in rhinovirus (RV)-positive and negative cases in the validation dataset. RV-positive: area under the curve (AUC) 0.84 (95\% CI 0.06, p<0.001); sensitivity $84.0 \%$; specificity 75.9\%. RV-negative: AUC 0.67 (95\% CI 0.06, p=0.005); sensitivity 62.9\%; specificity $65.6 \%$. 
TABLE 2 Electronic nose 1 validation set: performance characteristics of volatile organic compound analysis for children with lower respiratory tract symptoms

\begin{tabular}{|c|c|c|c|c|c|c|}
\hline & Mean AUC $(95 \% \mathrm{CI})$ & p-value & Sensitivity & Specificity & +LR & $-\mathrm{LR}$ \\
\hline \multicolumn{7}{|l|}{ Rhinovirus positive } \\
\hline Acute infection" & $0.77(0.07)$ & $<0.001$ & 73.0 & 96.0 & 18.25 & 0.28 \\
\hline \multicolumn{7}{|l|}{ Rhinovirus negative } \\
\hline Acute infection & $0.81(0.05)$ & $<0.001$ & 75.6 & 71.4 & 2.64 & 0.34 \\
\hline Recovered" & $0.67(0.06)$ & 0.005 & 62.9 & 65.6 & 1.83 & 0.57 \\
\hline
\end{tabular}

Sensitivities, specificities, and positive $(+L R)$ and negative likelihood ratios $(-L R)$ are reported for the respective optimum cut-points. Symptomatic infection was defined as the presence of expiratory wheeze as assessed by auscultation. Asymptomatic infections were defined as children never experiencing respiratory symptoms severe enough to consult a general practitioner. Recovered infections were defined as children with confirmed wheeze who were tested again after resolution of their first symptomatic infection. AUC: area under the curve. ${ }^{\#}$ : symptomatic versus asymptomatic; " : ex-symptomatic versus asymptomatic.

1000 random classifications and minimised the risk of false-positive results by reproducing the results with a second device. Finally, we used state-of-the-art, multiplex, quantitative PCR analysis in order to optimise virus identification in both oro- and nasopharyngeal samples [23, 27].

Our study has some limitations, which are largely implicit to its design. Firstly, $75 \%$ of the children with a symptomatic infection did not meet the criteria for a symptom-free period of $\geqslant 1$ week, 6 weeks after their primary episode due to recurrent or prolonged symptoms. This required their symptom-free re-assessment to be postponed. Due to the serial nature of infections with respiratory pathogens in children, it is not unlikely that some of them experienced another respiratory tract infection before being re-assessed [3]. We, however, deliberately chose only to reassess children when asymptomatic rather than after a fixed time period. The latter would have resulted in a mixed population of symptomatic and asymptomatic children, which grossly affects exhaled VOC as shown by the present data.

Secondly, 25\% of the children experienced multiple simultaneous infections, which have been observed to be associated with more severe illness [3]. It cannot be excluded that some of the children were symptomatic in response to one of the co-infected viruses rather than the rhinovirus. This may have biased interpretation of rhinovirus-associated changes in VOC profiles. Unfortunately, differentiation of effects by each of the 14 respiratory viruses and their combinations on exhaled markers would have required a far greater sample size, and reaches beyond the objective of the present study because their association with development of asthma is less pronounced in a general practice population.

The asymptomatic children were significantly older than the symptomatic children. This was due to the fact that children experiencing LRTS after being assessed as a control were excluded from this arm of the study. Subsequently, a novel, and therefore older, control had to be recruited. We believe this approach strengthened the study despite the potential age bias introduced due to the ageing of the controls. Furthermore, we did not observe that age affected the VOC profile despite the fact that airway dead space volume increases with age [28]. Additionally, VOC profiles were not significantly affected by the use of ICS or $\beta_{2}$-agonists. Finally, an effect of environmental smoke exposure and sex on the VOC profile cannot be excluded. As these latter two did not differ significantly between study groups, these are unlikely to affect our outcome.

eNose assessment allows probabilistic analysis and classification of subjects, but is principally not suited to identify individual exhaled molecular constituents [29]. The exact origins of the VOC differentiating symptomatic and asymptomatic children are unknown but most likely result from a combination of airway obstruction [30], an increase in oxidative stress, changes in the microcirculation and the hosts immune response [31]. These compounds are likely to have both pulmonary and systemic origins. Using gas chromatography-mass spectrometry (GC-MS), it appears that preschool wheezing is associated with mostly hydrocarbon-based exhaled VOC [16]. These closely resemble, but are not identical to, those that have been associated with asthma [17, 32]. Thus far, there is little knowledge on virus-associated VOC [33]. These are likely to be dependent on pathogen-host interactions, which may explain why symptomatic and asymptomatic children demonstrated different VOC profiles even when suffering from an identical infection (rhinovirus).

Remarkably, VOC profiles did not normalise in children with rhinovirus-induced wheeze even after recovery. This does not, however, demonstrate causality, as these findings may be interpreted in two ways. 
First, children who wheeze in response to rhinovirus may have a different pre-existing, biological host response compared with those who remain asymptomatic in the presence of rhinovirus in the airways. Second, rhinovirus itself may induce a prolonged inflammatory response in those children with rhinovirus-induced wheeze [4] possibly mediated through epigenetic changes [34]. Irrespective of the exact mechanism histological studies have shown that children with respiratory wheeze already exhibit histological findings suggestive of (developing) asthma [11]. Furthermore, a recent paper from the Childhood Origins of Asthma and Copenhagen Studies on Asthma in Childhood cohorts demonstrated that children who experience rhinovirus-induced wheeze and having a genetic variant of the 17q21 locus have a marked up regulation of ORMDL3 and GSDMB, and an impressive odds ratio of 26.1 for the development of asthma [35]. We can speculate that such genotype-environment interactions may contribute to the differences in VOC profiles after symptomatic recovery established in this study.

Our study presents the potential of noninvasive biomarkers in studying a high-risk phenotype in the development of asthma. In view of the established accuracy, the clinical relevance of our data is not represented by potentially using an eNose as a diagnostic tool for rhinovirus-induced wheeze. However, our data suggest that analysis of VOC can be used as a noninvasive measure of the host response to viral infection both during acute symptoms and thereafter. Even though the mechanisms underlying the 10-fold increased risk [6] of developing asthma after rhinovirus-associated wheeze remains to be established [5, 36], these findings do indicate that this group of children may already be captured by an exhaled biomarker fingerprint. These results may therefore be in line with recent findings showing that exhaled biomarkers correlate with inflammatory subphenotype (sputum eosinophils) in asthma [17, 18]. Longitudinal follow-up of the children in the present study, which is currently on-going, is necessary to establish the long-term predictive potential of exhaled markers for the development of asthma, as recently established for GC-MS [37].

In conclusion, an acute episode of respiratory wheeze in preschool children affects exhaled VOC. This appears to be a prolonged effect in children with rhinovirus-induced wheeze. Therefore, this composite, exhaled biomarker fingerprint may reflect differences in airway pathophysiology underlying these children's 10-fold increased risk of developing asthma. Our data raise the hypothesis that exhaled VOC have potential as a noninvasive method of inflammometry in the monitoring of early signs of asthma.

\section{Acknowledgments}

The authors would like to thank all children and parents participating in the EUROPA study whom enable us to do our research.

\section{References}

1 Henderson J, Granell R, Heron J, et al. Associations of wheezing phenotypes in the first 6 years of life with atopy, lung function and airway responsiveness in mid-childhood. Thorax 2008; 63: 974-980.

2 Morgan WJ, Stern DA, Sherrill DL, et al. Outcome of asthma and wheezing in the first 6 years of life: follow-up through adolescence. Am J Respir Crit Care Med 2005; 172: 1253-1258.

3 Jartti T, Lee WM, Pappas T, et al. Serial viral infections in infants with recurrent respiratory illnesses. Eur Respir J 2008; 32: 314-320.

4 Schneider D, Hong JY, Popova AP, et al. Neonatal rhinovirus infection induces mucous metaplasia and airways hyperresponsiveness. J Immunol 2012; 188: 2894-2904.

5 Kieninger E, Fuchs O, Latzin P, et al. Rhinovirus infections in infancy and early childhood. Eur Respir J 2013; 41: 443-452.

6 Jackson DJ, Gangnon RE, Evans MD, et al. Wheezing rhinovirus illnesses in early life predict asthma development in high-risk children. Am J Respir Crit Care Med 2008; 178: 667-672.

7 Fouzas S, Brand PLP. Predicting persistence of asthma in preschool wheezers: crystal balls or muddy waters? Pediatr Respir Rev 2013; 14: 46-52.

8 Kotaniemi-Syrjänen A, Malmberg LP, Malmström K, et al. Factors associated with elevated exhaled nitric oxide fraction in infants with recurrent respiratory symptoms. Eur Respir J 2013; 41: 189-194.

9 Caudri D, Wijga AH, Hoekstra MO, et al. Prediction of asthma in symptomatic preschool children using exhaled nitric oxide, Rint and specific IgE. Thorax 2010; 65: 801-807.

10 Pijnenburg MWH, De Jongste JC. Exhaled nitric oxide in childhood asthma: a review. J Allergy Clin Immunol 2008; 38: 246-259.

11 Saglani S, Payne DN, Zhu J, et al. Early detection of airway wall remodeling and eosinophilic inflammation in preschool wheezers. Am J Respir Crit Care Med 2007; 176: 858-864.

12 Wheelock CE, Goss VM, Balgoma D, et al. Application of 'omics technologies to biomarker discovery in inflammatory lung diseases. Eur Respir J 2013; 42: 802-825.

13 Dragonieri S, Schot R, Mertens BJ, et al. An electronic nose in the discrimination of patients with asthma and controls. J Allergy Clin Immunol 2007; 120: 856-862.

14 Fens N, Roldaan AC, van der Schee MP, et al. External validation of exhaled breath profiling using an electronic nose in the discrimination of asthma with fixed airways obstruction and chronic obstructive pulmonary disease. Clin Exp Allergy 2011; 41: 1371-1378.

15 Robroeks CM, van Berkel JJ, Jöbsis Q, et al. Exhaled volatile organic compounds predict exacerbations of childhood asthma in a 1-year prospective study. Eur Respir J 2013; 42: 98-106. 
16 Van de Kant KDG, van Berkel JJ, Jöbsis Q, et al. Exhaled breath profiling in diagnosing wheezy preschool children. Eur Respir J 2013; 41: 183-188.

17 Ibrahim B, Basanta M, Cadden P, et al. Non-invasive phenotyping using exhaled volatile organic compounds in asthma. Thorax 2011; 66: 804-809.

18 Van der Schee MP, Palmay R, Cowan JO, et al. Predicting steroid responsiveness in patients with asthma using exhaled breath profiling. Clin Exp Allergy 2013; 43: 1217-1225.

19 Keil T, McBride D, Grimshaw K, et al. The multinational birth cohort of EuroPrevall: background, aims and methods. Allergy 2010; 65: 482-490.

20 Chalut DS, Ducharme FM, Davis GM. The Preschool Respiratory Assessment Measure (PRAM): a responsive index of acute asthma severity. J Pediatr 2000; 137: 762-768.

21 Juniper EF, O'Byrne PM, Guyatt GH, et al. Development and validation of a questionnaire to measure asthma control. Eur Respir J 1999; 14: 902-907.

22 Asher MI, Keil U, Anderson HR, et al. International study of asthma and allergies in childhood (ISAAC): rationale and methods. Eur Respir J 1995; 8: 483-491.

23 De Vries M, Deijs M, Canuti M, et al. A sensitive assay for virus discovery in respiratory clinical samples. PLoS One 2011; 6: e16118.

24 Schee MP Van Der, Fens N, Brinkman P, et al. Effect of transportation and storage using sorbent tubes of exhaled breath samples on diagnostic accuracy of electronic nose analysis. J Breath Res 2013; 7: 016002.

25 Broadhurst D, Kell D. Statistical strategies for avoiding false discoveries in metabolomics and related experiments. Metabolomics 2006; 4: 171-196.

26 Sung J, Wang Y, Chandrasekaran S, et al. Molecular signatures from omics data: from chaos to consensus. J Biotechnol 2012; 7: 946-957.

27 Turchiarelli V, Schinkel J, Molenkamp R, et al. Repeated virus identification in the airways of patients with mild and severe asthma during prospective follow-up. Allergy 2011; 66: 1099-1106.

28 Miekisch W, Schubert JK, Noeldge-Schomburg GFE. Diagnostic potential of breath analysis-focus on volatile organic compounds. Clin Chim Acta 2004; 347: 25-39.

29 Röck F, Barsan N, Weimar U, et al. Electronic nose: current status and future trends. Chem. Rev 2008; 108 : 705-725.

30 Boshier PR, Priest OH, Hanna GB, et al. Influence of respiratory variables on the on-line detection of exhaled trace gases by PTR-MS. Thorax 2011; 66: 919-920

31 Buszewski B, Kesy M, Ligor T, et al. Human exhaled air analytics: biomarkers of diseases. Biomed Chrom 2007; 21: $553-566$.

32 Dallinga JW, Robroeks CM, van Berkel JJ, et al. Volatile organic compounds in exhaled breath as a diagnostic tool for asthma in children. Clin Exp Allergy 2010; 40: 68-76.

33 Bos LD, Sterk PJ, Schultz MJ. Volatile metabolites of pathogens - a systematic review. PLoS Pathog 2013; 9: e1003311.

34 McErlean P, Favoreto S, Costa FF, et al. Human rhinovirus infection causes different DNA methylation changes in nasal epithelial cells from healthy and asthmatic subjects. BMC Med Genomics 2014; 7: 37.

35 Calışkan M, Bochkov YA, Kreiner-Møller E, et al. Rhinovirus wheezing illness and genetic risk of childhood-onset asthma. New Engl J Med 2013; 368: 1398-1407.

36 Holt PG, Sly PD. Viral infections and atopy in asthma pathogenesis: new rationales for asthma prevention and treatment. Nat Med 2012; 18: 726-735.

37 Smolinska A, Klaassen EMM, Dallinga JW, et al. Profiling of volatile organic compounds in exhaled breath as a strategy to find early predictive signatures of asthma in children. PLoS One 2014; 9: e95668. 